\title{
Epidemiology of canine heartworm (Dirofilaria immitis) infection in domestic dogs in Ontario, Canada: Geographic distribution, risk factors and effects of climate
}

\author{
Erin McGill, ${ }^{1}$ Olaf Berke, ${ }^{1}$ Andrew S. Peregrine, ${ }^{2}$ J. Scott Weese ${ }^{2}$ \\ ${ }^{1}$ Department of Population Medicine, Ontario Veterinary College, University of Guelph; ${ }^{2}$ Department of \\ Pathobiology, Ontario Veterinary College, University of Guelph, Canada
}

\begin{abstract}
Dirofilaria immitis is the causal agent of heartworm, a mosquito-borne parasite that primarily infects domestic and wild canids. The infection is endemic in parts of Canada, and Ontario has been identified as the province where the majority of heart-
\end{abstract}

Correspondence: Erin McGill, Department of Population Medicine, Ontario Veterinary College, University of Guelph, 553 Edinburgh Road South, Guelph (ON), N1G 4E7 Canada.

Tel.: 1.613.720.9987.

E-mail: erin.mcgill@canada.ca

Key words: Dirofilaria immitis; Risk factors; Prevalence; Geospatial; Canada.

Acknowledgements: the authors would like to thank IDEXX Laboratories Canada Corp and Dr. Dan McKenney from the ANUSPLIN Project - Forest Resources - Natural Resources Canada for providing data and the Zoetis Investment in Innovation Fund for providing funding for this project.

Contributions: EM, OB, study objectives, data interpretation; EM, data cleaning, data analysis and manuscript writing; JSW secured laboratory data from IDEXX Laboratories Canada Corp.; OB, JSW, ASP, critical feedback and manuscript reviewing.

Conflict of interest: the authors declare no potential conflict of interest.

Funding: the work was supported by a Zoetis Investment in Innovation grant.

Conference presentation: part of this paper was presented at the International Society for Companion Animal Infectious Diseases, 2018 September 30-October 3, Portland, Oregon, USA.

Received for publication: 18 September 2018.

Revision received: 26 October 2018.

Accepted for publication: 12 November 2018.

(C) Copyright E. McGill et al., 2019

Licensee PAGEPress, Italy

Geospatial Health 2019; 14:741

doi:10.4081/gh.2019.741

This article is distributed under the terms of the Creative Commons Attribution Noncommercial License (CC BY-NC 4.0) which permits any noncommercial use, distribution, and reproduction in any medium, provided the original author(s) and source are credited. worm infections occur. Test results for blood samples submitted by veterinary clinics for the years 2007-2016 were used to conduct a spatial risk analysis of heartworm among domestic dogs in Ontario. The geographic extent of the apparent heartworm prevalence was examined through smoothed choropleth maps for all 49 census division regions. Furthermore, the regions were assessed for local clusters in apparent prevalence using the flexible spatial scan statistic. Three clusters were found and located in western, southern and eastern Ontario, respectively. A spatial Poisson regression model for heartworm prevalence among pet dog populations in southern Ontario census divisions was fit to determine the association between human population size, heartworm development units (HDUs), climate moisture index (CMI), precipitation and directions, east or north, with heartworm infection. The model identified the spatial distribution of HDUs and CMI as positively associated with heartworm infection and therefore important predictors of the infection. In contrast, human population size, increasing northern latitude and drier areas were negatively associated with heartworm infection. The east direction and precipitation were not significant.

\section{Introduction}

The parasite Dirofilaria immitis, commonly known as heartworm, primarily infects dogs but can also infect felids, wild canids and, though very rarely, humans (Vezzani and Carbajo, 2006). Domestic dogs and wild canids serve as the primary reservoirs (Ciucă et al., 2016) with mosquitoes the only known intermediate host and vector of $D$. immitis (Slocombe et al., 1989).

In Ontario, Canada there are at least 22 mosquito species reported as potential vectors of $D$. immitis (Ludlam et al., 1970). On the basis of historical data, Aedes vexans, Culex pipiens-restuans and Mansonia perturbans are the most common and widespread in southern Ontario, capable of supporting D. immitis larvae development to the infective stage (Ernst and Slocombe, 1984; Slocombe et al., 1989). Ae. vexans is the most common vector in the fall and survives into at least mid-September; its life span is approximately 30 days (Slocombe et al., 1989). Ae. stimulans can be found as early as mid-May and can live into the summer months (Slocombe et al., 1989).

The threshold temperature to allow for parasite maturation to the infective stage within the mosquito is $14^{\circ} \mathrm{C}$; there is a linear relationship between rate of development of the parasite and temperatures from $18^{\circ} \mathrm{C}$ to $34^{\circ} \mathrm{C}$ (Fortin and Slocombe, 1981). The amount of heat required for larval development to the infective stage is 130 heartworm development units (HDU) (Slocombe et al., 1989), which is defined as the cumulative sum of daily aver- 
age degrees centigrade above the $14^{\circ} \mathrm{C}$ threshold temperature within a period of 30 days (Slocombe et al., 1989; Knight and Lok, 1998). In Canada, warmer temperatures are generally first observed in southern Ontario compared to other areas in the province and country. Based on weather data from 1957-1986 collected from Windsor, Ontario the transmission period for Canada is considered June $1^{\text {st }}$ to October $9^{\text {th }}$ (Slocombe et al., 1989). However, the wide range of climate across Canada means that the true risk period varies geographically. Historically, the start of transmission was calculated by summing daily HDUs from May $6^{\text {th }}$ (date of earliest blood feeding for Ae. stimulans) until 130 HDUs are achieved (Slocombe et al., 1989). The end of the transmission period has historically been calculated as the last day in the year when 130 HDUs can be reached within 30 days (based on the life span of Ae. vexans a late season vector) (Slocombe et al., 1989). Some mosquitoes are capable of overwintering; however, it is assumed that the effect of overwintering mosquitoes on the overall heartworm transmission pattern is negligible (Knight and Lok, 1998). On the basis of the aforementioned transmission season, the recommendation for preventive medication in Canada is July $1^{\text {st }}$ to November $1^{\text {st }}$, as seasonal preventive treatment is to begin within the first month of the start of the transmission season and continue until the first of the month following the end of the transmission period (Graham et al., 2012). As a conservative approach, the recommended start date for prophylaxis is June $1^{\text {st }}$. Macrocyclic lactones, the drug family used for preventive medication, are marketed as oral (ivermectin and milbemycin oxime), topical (moxidectin and selamectin) and subcutaneous injectable (slow-release moxidectin-impregnated lipid microspheres) products, which all have one month's reach-back activity (Graham et al., 2012).

Climate change has been implicated as a possible factor for increases in heartworm prevalence that have been observed in the United States in dogs, due to faster development to the infective larval stage in mosquitoes (Ledesma and Harrington, 2011). A temperature increase can also lead to the emergence of the parasite in new areas, as mosquitoes are able to extend their habitat range (Sassnau et al., 2014). Furthermore, climate change can lengthen the annual transmission season by extending the periods when temperatures are sufficient for parasite development (Morchón et al., 2012). It is possible that climate change has impacted the heartworm infection prevalence among domestic Ontario dogs.

Many factors influence transmission of heartworm including those that impact mosquito populations. Precipitation, vegetation and moisture can impact breeding, consequentially impacting $D$. immitis transmission (Alho et al., 2014). Mosquitoes also require heat; large cities may produce heat islands creating more favourable conditions for mosquito breeding compared to rural environments (Morchón et al., 2012; Herrin et al., 2017). However, on the basis of data from the United States, Wang et al. (2014) found that heartworm prevalence in dogs decreased with increasing human population density and elevation, a possible explanation being the difference in humidity and temperature at higher elevations. It is not known how factors, such as precipitation, which impact the mosquito population, may impact heartworm infection among domestic dogs in Ontario.

The majority of testing of dogs for heartworm in Canada occurs in Ontario (Klotins et al., 2000). The spatial distribution of heartworm infection among dogs in Ontario was most recently assessed from 2013-2014; however, the spatial pattern over a longer time period has not been determined (Herrin et al., 2017). Although historic areas of risk are known, those regions may have experienced changes. Furthermore, the association between heartworm infection and risk factors such as HDUs, precipitation, moisture and urban density have not been examined in Ontario. The objectives of this study were therefore, firstly, to determine the prevalence of heartworm in dogs at the Ontario census division level and visualize its geographic distribution by choropleth mapping. Secondly, to assess the presence of spatial clusters of heartworm infection in dogs in Ontario using the flexible scan test. And thirdly, to assess the association between environmental predictors including HDUs, precipitation, moisture index and human population size and heartworm infection prevalence in companion dogs using a spatial Poisson regression model.

\section{Materials and Methods}

The dataset for this study consisted of $D$. immitis antigen tests performed at a commercial diagnostic laboratory (IDEXX laboratories) and in veterinary clinics from March 2007 to July 2016. Three different antigen tests were used during the study period: the SNAP 3Dx antigen test for the years 2007 to 2012, the SNAP 4Dx antigen test for 2011 to 2012, and the SNAP 4Dx Plus Test for 2012 to 2016. SNAP 3Dx tested for D. immitis, Borrelia burgdorferi and Ehrlichia canis. SNAP 4Dx and 4Dx Plus tested for $D$. immitis, B. burgdorferi, E. canis, Anaplasma phagocytophilum and $A$. platys. Data consisted of test result, dog age and postal code of the veterinary clinic where the sample was collected, or the test was performed.

The dataset was cleaned by removing duplicate sample submission numbers and non-canine species e.g. felines. Test results that had not been recorded as 0 (negative) or 1 (positive) were removed. The age of the dog were reported in months; dogs younger than 6 months of age (minimum age the antigen test would be able to detect heartworm) and older than 240 months (20 years) were removed.

Natural Resource Canada (https:/www.nrcan.gc.ca/home) supplied HDUs and climate normals for the centroid of all 49 Ontario census divisions based on thin plate spline smoothing algorithms to develop spatially continuous climate models using weather station data (Natural Resources Canada, 2018). The centroid locations were applied to the grid model to provide the climate normals for 1980 to 2010 for that location, including the mean moisture index and mean precipitation. The mean climate moisture index (CMI) was provided in $\mathrm{cm}$ and mean precipitation in mm. Natural Resource Canada also applied the HDU model (Slocombe et al., 1989) to observed recorded daily temperatures using the grid model for the census division centroids and provided HDU data at a weekly level for the years 2005 to 2016 . The weekly HDU data were then used to determine the transmission season and cumulative HDUs for that season. The start of transmission was defined as the accumulation of a minimum of 130 HDUs within 4 weeks (using the weekly data provided), roughly the lifespan of a mosquito (Fortin and Slocombe, 1981; Sassnau et al., 2014). End of transmission was the last week that 130 HDUs could be accumulated within 4 weeks. Annual cumulative HDUs were determined as the sum of HDUs for the transmission season of each year. These annual cumulative HDUs were then aggregated at the census division level over the years 2005 to 2016 (i.e. the years prior to and concurrent with heartworm testing).

Information regarding human population size and dwelling number was retrieved from the 2011 census conducted by Statistics 
Canada (2011). The prevalence of companion dog heartworm infections was examined at the Ontario census division level. The six-digit postal codes of the veterinary clinics (as supplied with the diagnostic test results) were matched to their Multiple Enhanced Postal Code (MEP) identification number (DMTI Spatial Inc., 2018). The MEPs were then matched to the corresponding census division number using a postal code to census division translation table (DMTI Spatial Inc., 2018). Both current and retired MEPs were used because the data spanned from 2007 to 2016. Duplicate postal codes were combined, and clear typographical errors based on the pattern followed by Canadian postal codes (e.g. 0, O) were corrected. Reports without a postal code were excluded. Reports that were labelled Ontario but had a non-Ontario postal code were also excluded. To visually display the heartworm prevalence estimates across Ontario, a boundary map file of the province's 49 census divisions was retrieved from Statistics Canada (2016) for the 2011 census year; the census division boundaries were extracted from the respective Canada census division boundary file. The boundary file projection was then converted from Mercator to Universal Transverse Mercator Zone $17 \mathrm{~N}$ for better visualization of the province's landmass.

The denominator of the census division prevalence estimates, i.e. the number of tests performed, was not consistent across the divisions. Thus, empirical Bayesian smoothing was used to internally standardize the differences in sample size (Berke, 2004; Beyer et al., 2012). The annual prevalence was smoothed for each census division and the smoothed prevalence estimates were aggregated over time to generate a single choropleth map for the years 2006 to 2016 . The choropleth map visualized the geographic variation in heartworm prevalence and any cluster of census divisions with a higher than expected prevalence. A flexible spatial scan test was applied to determine the location of probable clusters and estimate the relative risk of prevalence for the respective clusters compared to regions outside the cluster (Tango and Takahashi, 2012). The maximum number of census divisions used for the flexible scan test was set as 7 regions to ensure the populations assessed did not constitute more than $50 \%$ of the Ontario population. The significance of the test result was assessed using estimated p-values based on 999 Monte Carlo simulations. The clustering of infections, i.e. strength of spatial correlation of heartworm infection among domestic dogs, was tested through the Moran's I correlation coefficient using the queen spatial structure where all touching polygons are considered neighbours (Assunção and Reis, 1999; Waller and Gotway, 2004).

In additional work, a model was built to examine the risk of heartworm infection among domestic dogs in Ontario. Initially, a Generalized Linear Model was used to assess putative risk factors. To correct for overdispersion, a Generalized Linear Mixed Model (GLMM) of the Poisson family with an exponential spatial correlation structure was fit using the Penalized Quasi-Likelihood (PQL) estimation method. The following factors that have been shown to have a positive association with heartworm infection risk in dogs were included in this model building process to assess their effects in the current study population: average dog age, human population size (number of persons), human population density (per one $\mathrm{km}^{2}$ ), agricultural regions, number of human dwellings, CMI, precipitation and HDUs. The potential for a spatial trend in east/west and north/south directions was assessed using polynomials in the centroid coordinates of the census division. Collinearity of the quantitative predictor variables was assessed by the variance inflation factors (VIF), where VIF $>10$ was considered to indicate multicollinearity (Dohoo et al., 2003). The spatial GLMM model fit was assessed by examining the predictive properties, linearity and normality. The predictive properties of the model were assessed through a plot that examined the predicted and observed prevalence. Normalized residuals were evaluated with a scatterplot of the residuals against the quantitative predictors of the model and a line of best fit. A QQ-plot was used to check the normality of the normalized residuals. A circular scan test under the normal model was performed on the normalized residuals to detect any potential clusters not explained by the predictors in the regression model (Kulldorff et al., 2009). The spatial scan test was applied for both high and low residual values; significance of the test results was assessed using estimated p-values based on 999 Monte Carlo simulations.

ArcGIS (ESRI, Redlands, CA, USA) was used for conversion of postal codes to the census division level (ESRI, 2011). R and RStudio were used to generate the choropleth maps, and to perform the flexible scan test (smerc package) and for the spatial regression model analysis (RStudio Team, 2016; R Core Team, 2017). Normalized residual analysis was conducted in SaTScan (SaTScan, 2018). A significance level of $\alpha=0.05$ was applied for all analyses, unless otherwise indicated.

\section{Results}

A total of 660,946 Ontario dog test results were included in this study of which 629,720 were from dogs that inhabited southern Ontario census divisions. There were 762 test positive dogs in Ontario between 2007 and 2016; 722 lived in southern Ontario. The total Ontario heartworm prevalence over 2007 to 2016 was $0.12 \%(95 \%$ CI: $0.10,0.12)$, and ranged over time from $0.32 \%$ (95\% CI: $0.20,0.52)$ in 2008 to $0.13 \%$ (95\% CI: $0.11,0.15)$ in 2015. With respect to geographic variation, Haldimand-Norfolk census division had the highest prevalence over 2007 to 2016 with $0.64 \%$ (95\% CI: $0.55,0.75)$ followed by Chatham-Kent at $0.59 \%$ (95\% CI: $0.49,0.71)$ and Rainy River at $0.50 \%$ (95\% CI: 0.33 , 0.75). The ten census divisions of Prince Edward, Kawartha Lakes, Dufferin, Muskoka, Haliburton, Parry Sound, Manitoulin, Sudbury, Timiskaming, Cochrane and Algoma had no positive test results from 2007 to 2016. The sample size from 2007 to 2016 increased on by a factor of $\sim 1.5$ each year. The number of positive test results also increased from 16 in 2008 to 176 in 2015; the years 2007 and 2016 were partially sampled years.

The choropleth map of the smoothed heartworm prevalence for the 49 Ontario census divisions from 2007 to 2016 is shown in Figure 1; southern Ontario data are presented at higher resolution in Figure 2. There were three hotspots for heartworm infection identified by the flexible scan test: southern Ontario (HaldimandNorfolk, Oxford, Elgin, Chatham-Kent and Lambton), western Ontario (Rainy River), and eastern Ontario (Lanark and Renfrew) (Figures 1 and 2). Each hotspot is outlined in a different colour for identification and was significant at the $\alpha=0.05$ level. The cluster in southern Ontario had 328 positive test results, where the expected number was 77 , and the prevalence risk ratio (PRR) of canine heartworm infection of the cluster was 6.7 , i.e. the risk in the cluster was almost 7 times greater than the risk in the rest of Ontario. Rainy River had 22 positive test results from 2007 to 2016; the expected number was 5 test positives and the $P R R=4.3$. Eastern Ontario had 36 positive test results from 2007 to 2016; the expected number was 14 . The PRR was lowest in this eastern hotspot at 
2.6. The value of Moran's I correlation coefficient for Ontario was $0.17, \mathrm{P}=0.008$; indicating the presence of spatial clustering.

The final spatial Poisson regression model included several risk factors for heartworm infection among domestic dogs and is summarized in Table 1. Human population size had a protective effect on heartworm infection prevalence; as the number of persons within a census division increased, the risk of heartworm infection in dogs decreased $(\mathrm{P}=0.0074$; $\mathrm{PRR}=0.63$ with a population of 500,000 and decreased to 0.40 with a population of $1,000,000$ people per census division). There was a positive association between HDUs and heartworm prevalence $(\mathrm{P}=0.006)$; the PRR doubled for each increase of 100 HDUs within a transmission season. There was an interaction effect between CMI and increasing north latitude $(\mathrm{P}=0.01)$ on the heartworm prevalence. Neither variable was associated with the outcome on its own; however, their interaction was an important model component and indicated an association between heartworm infection and CMI. Heartworm prevalence infection risk increased with increasing CMI conditional on the interaction effect.

Precipitation was not significant but was kept in the model because it has been cited in the literature as a positive risk factor (Alho et al., 2014; Wang et al., 2014). It also had a large effect within the model, as the prevalence risk of heartworm doubled for each additional $5 \mathrm{~mm}$ of rain, meaning that in areas that experience greater amounts of precipitation there may be an increased risk of heartworm.

Dog age was originally included in the spatial regression model for a basic epidemiological understanding of population dynamics; it was found to have a negative association with heartworm infection (i.e. older dogs had a reduced risk of infection). However, this was not significant, and the effect was very small; thus, it was excluded. Agricultural regions were also originally included in the model but were found to be not significant and without a large effect. Human population density was cited as a risk factor for dog populations in the United States (Brown et al., 2012; Wang et al., 2014); however, population density (per one kilometer ${ }^{2}$ ) was not associated with heartworm infection in this study. Lastly, the total number of private human dwellings per census division was initially considered for inclusion, but when assessed for collinearity was highly influenced by population size and excluded.

The scatterplot of the predicted versus observed prevalence of heartworm infection in dogs estimated by census division (Figure 3) did not indicate a lack of fit for the regression model.
Furthermore, the circular spatial scan test applied to the normalized deviance residuals did not indicate the presence of any residual cluster. Thus, the predictors in the regression model explained the observed clusters (Figure 3).

\section{Discussion}

Southern Ontario has historically been the focus of heartworm prophylaxis and concern for this infection in dogs in Canada (Klotins et al., 2000). The results from this study, by looking at data over 2007 to 2016, show that in addition to the cluster in southern Ontario, there was also a cluster in northern Ontario, in Rainy River (Figure 1). Historically, northern Ontario did not have a heartworm cluster (Slocombe, 2011; Herrin et al., 2017). It is possible that veterinarians in Rainy River did not expect heartworm to occur that far north and therefore did not recommend a preventive medication to dog owners. A changing climate provides an alternative explanation for the emergence, or newly discovered, cluster of heartworm infection in Rainy River; warmer temperatures may have allowed mosquitoes and $D$. immitis to move further north (Genchi et al., 2011).

Climate change has been implicated in the changing tick distribution, including Ixodes scapularis the vector of B. burgdorferi; thus, Lyme disease has become a growing concern in Ontario (Greer et al., 2008; McPherson et al., 2017). More veterinarians are recommending testing for $B$. burgdorferi infection and the same diagnostic test kit is used for the detection of heartworm. It could be that increased testing for tick-borne pathogens is leading to the detection of heartworm infections, which could explain the newly detected cluster in northern Ontario. The increasing sample size each year may account for more infections being detected. Furthermore, the proportion of tests occurring after heartworm season testing (April to June) increased from $24 \%$ in 2008 to $30 \%$ in 2015 (2007 and 2016 were partial years); suggesting the $D$. immitis positive results detected later in the year may have been accidental in testing for tick-borne pathogens.

The historic HDU model was built using weather data from 1957-1986 and research on Ontario mosquito species from the 1970 's and 80's; both of which may have experienced changes. It is important for further research to determine the mosquito species which are currently most common in Ontario, and elsewhere in Canada, and capable of supporting $D$. immitis development to the infective stage. The spatial model using the newly estimated HDUs

Table 1. Summary of the output from a spatial Generalized Linear Mixed Model Penalized Quasi-likelihood model for heartworm infection prevalence among dogs in southern Ontario over 2007 to 2016.

\begin{tabular}{lcccc} 
Variable & Coefficient Value & Variable Range* & P-value & \\
East $(\mathrm{km})$ & -0.0030 & $352.8-975.7$ & 0.1967 & 0.15 \\
North $(\mathrm{km})$ & 0.0088 & $4669.4-5058.0$ & 0.0866 & 30.55 \\
\hline Precipitation $(\mathrm{mm})$ & 0.0976 & $74.5-94.6$ & 0.0926 & 7.08 \\
CMI $(\mathrm{cm})$ & 31.97 & $-0.73-1.96$ & 0.0100 & 0.00 \\
\hline HDU & 0.0101 & $211.3-816.8$ & 0.0006 & 453.07 \\
Human population size & $-9.26 \times 10^{-7}$ & $17,026-2,615,060$ & 0.0074 & 0.09 \\
\hline I (north CMI) & -0.0066 & $-3408.7-9913.7$ & 0.0104 & 0.001 \\
\hline
\end{tabular}

PRR, prevalence risk ratio for the total variable; CMI, Climate moisture index; HDU, Heartworm development units (the amount of heat required for larval development to the infective stage (130 HDU). *Minimum and maximum value for that variable. 
supplied by Natural Resource Canada (2018) (based on the historic model) accurately predicted the heartworm prevalence observed from the data, which illustrated the validity of the HDU model.

The prevalence referred to in this paper is the estimated apparent prevalence of heartworm infection among domestic dogs in Ontario. The sensitivity and specificity of the 4Dx Plus Test for detecting $D$. immitis are $99.0 \%$ and $99.3 \%$, respectively (IDEXX, 2016). False positives and false negatives from the heartworm antigen tests are a limitation and influence estimation of the true heartworm risk among dogs; false positives lead to an over-estimation of the prevalence, while false negatives lead to an under-representation of the true prevalence. Thus, the positive predictive value using the overall Ontario prevalence of $0.11 \%$ for the study years 2007 to 2016 , and a test $99.0 \%$ sensitivity and $99.3 \%$ specificity, is $95 \%$, leaving $5 \%$ of test positives misclassified as positive. The negative predictive value estimated using the same values is $99.9 \%$, meaning the antigen tests used are better at classifying negative test results accurately than positive results. Berke and Waller (2010) showed that spatial data analyses for the detection of geographic/spatial patterns in infection occurrence are not seriously affected by diagnostic misclassification if the sample size is large in each region. However, what large sample sizes are is not specified (Berke and Waller, 2010). In the current study, large numbers of dogs were tested, overall, but heartworm is a rare event in Ontario which might be the reason that veterinarians in some census divisions did not detect cases.

The spatial Poisson regression model accurately predicted the observed census division heartworm prevalence from the laboratory dataset. Thus, variables that were included based on literature from Europe and the United States (Brown et al., 2012; Sassnau et

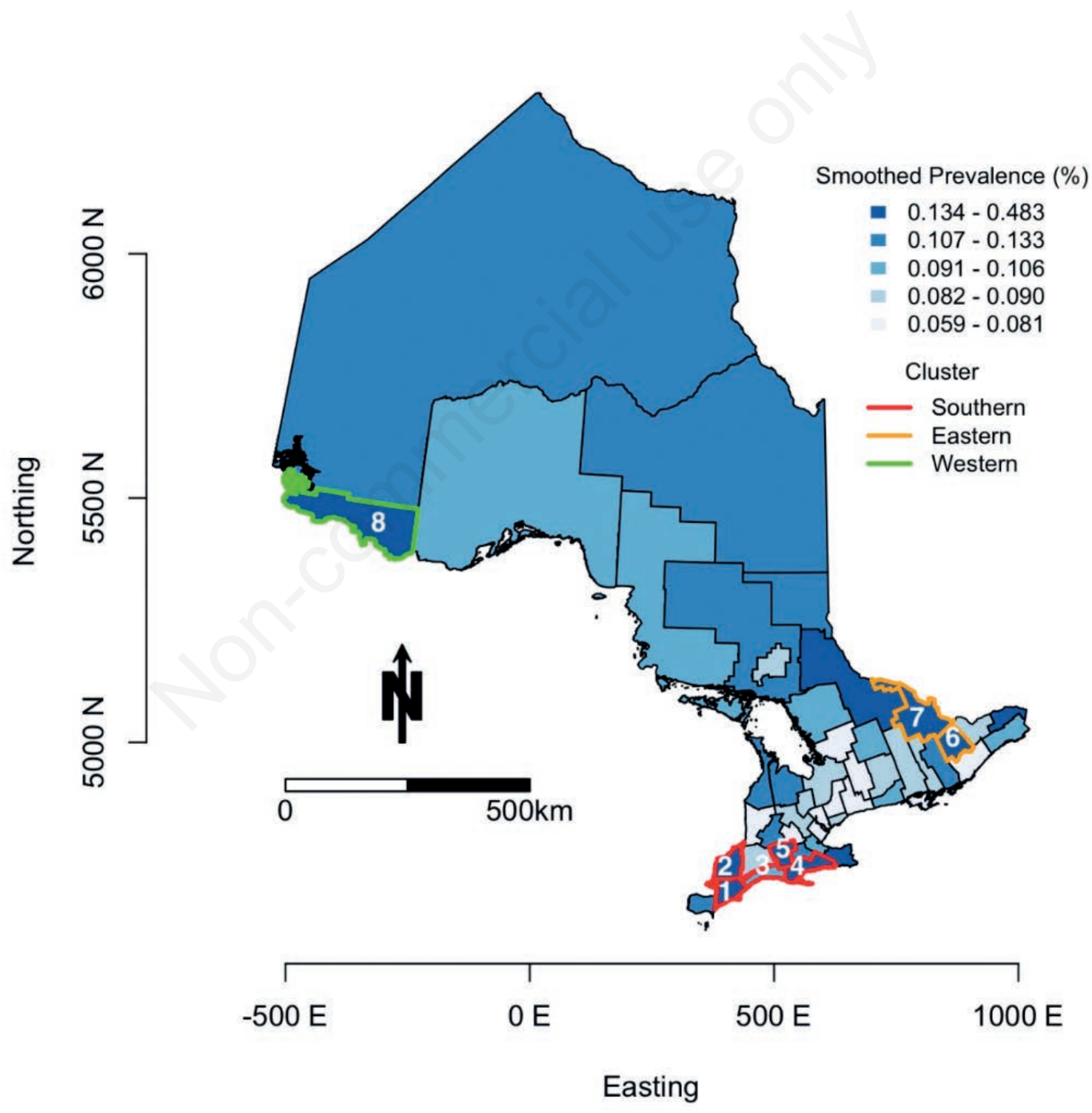

Figure 1. Choropleth map of all 49 Ontario census divisions with aggregated smoothed heartworm infection prevalence from 2007 to 2016 using laboratory data for all dogs tested. Red = primary cluster in southern Ontario (1- Chatham-Kent, 2- Lambton, 3- Elgin, 4Haldimand-Norfolk, 5- Oxford); Orange = secondary cluster in eastern Ontario (6- Lanark, 7- Renfrew); Green = other secondary cluster in western Ontario (8- Rainy River). 
al., 2014), were applicable to the Ontario population of dogs. The model initially was built for all 49 Ontario census divisions. However, the geography of Ontario (with extreme large northern regions that supplied few samples for testing as opposed to smaller regions in the south that supplied the majority of samples) required splitting-up the study area. Indeed, the PQL estimation algorithm for a model for the northern and southern Ontario heartworm prevalence combined would not converge. Since most of the Ontario human population and their domestic dogs inhabit southern Ontario, the model was built for this population only.

Limitations of this study include lack of information regarding repeated testing for individual dogs. The dataset included a postal code, but that was for the veterinary clinic location, not the residence of the dog. Lastly, travel history outside the province or the country, was not available for this study. To avoid spatial misclas- sification bias, the point data were aggregated to a census division level; any small discrepancies due to human error recording of postal codes should not have influenced the data interpretation because of the aggregation from point data to regional data.

As climate change continues and leads to warmer temperatures supportive of extrinsic development of D. immitis, it is important to determine areas of risk and be aware of vector spread into new environments. Historically, most heartworm cases in dogs were discovered in south-western Ontario (Klotins et al., 2000; Slocombe, 2011). However, the most southern census division of Essex, home to Windsor city (and the weather stations that supplied the data for the original HDU model for Ontario) was not included in the hotspot identified in this study. It is possible that there is a heightened awareness in that region which contributes to high prophylaxis and therefore the $\operatorname{dog}$ population is less susceptible to infection.

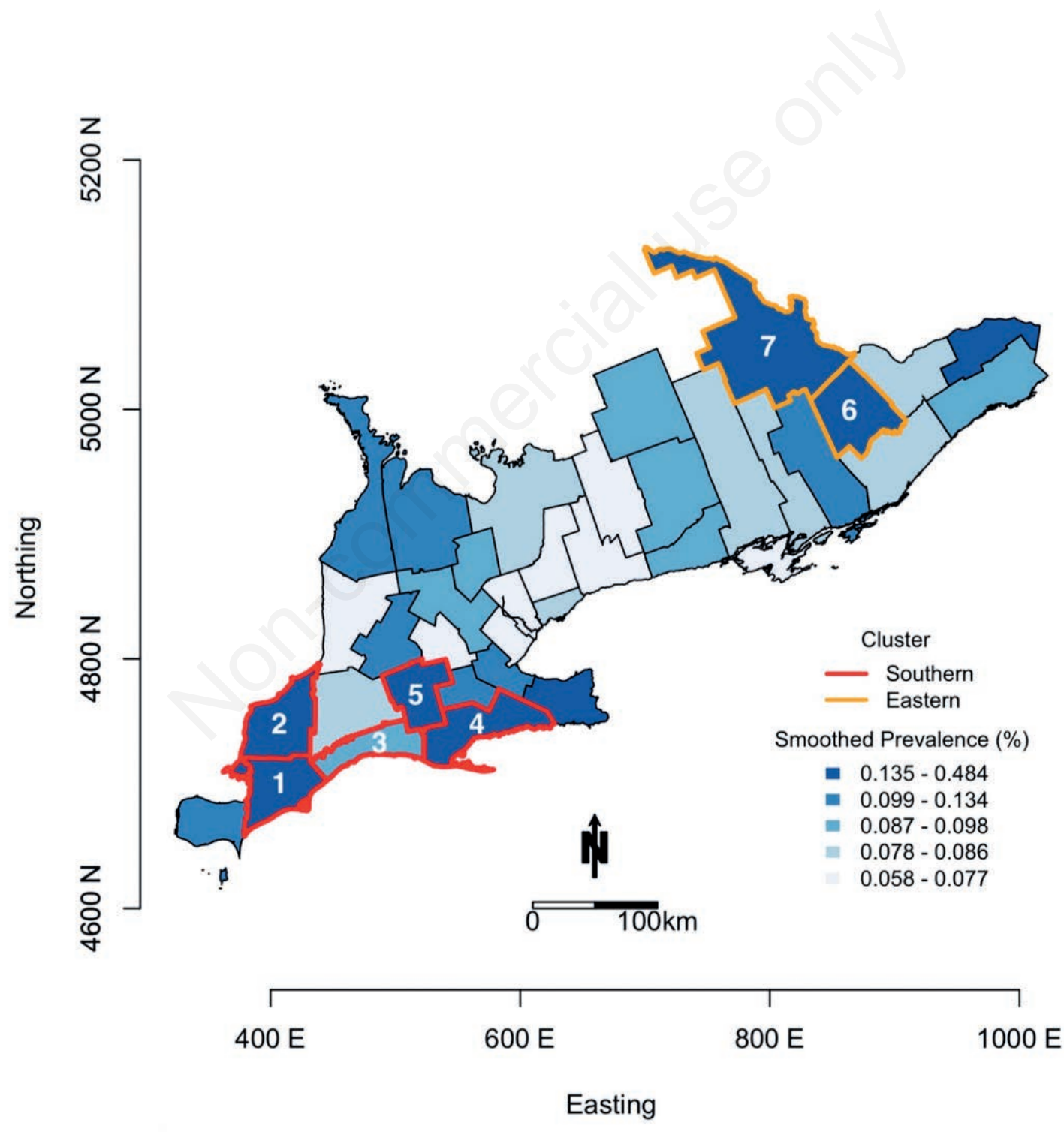

Figure 2. Choropleth map of the 37 census divisions in southern Ontario with aggregated smoothed heartworm infection prevalence from 2007 to 2016, using laboratory data for all dogs tested. Red = primary cluster in southern Ontario (1- Chatham-Kent, 2 Lambton, 3- Elgin, 4- Haldimand-Norfolk, 5- Oxford); Orange = secondary cluster in eastern Ontario (6- Lanark, 7- Renfrew). 


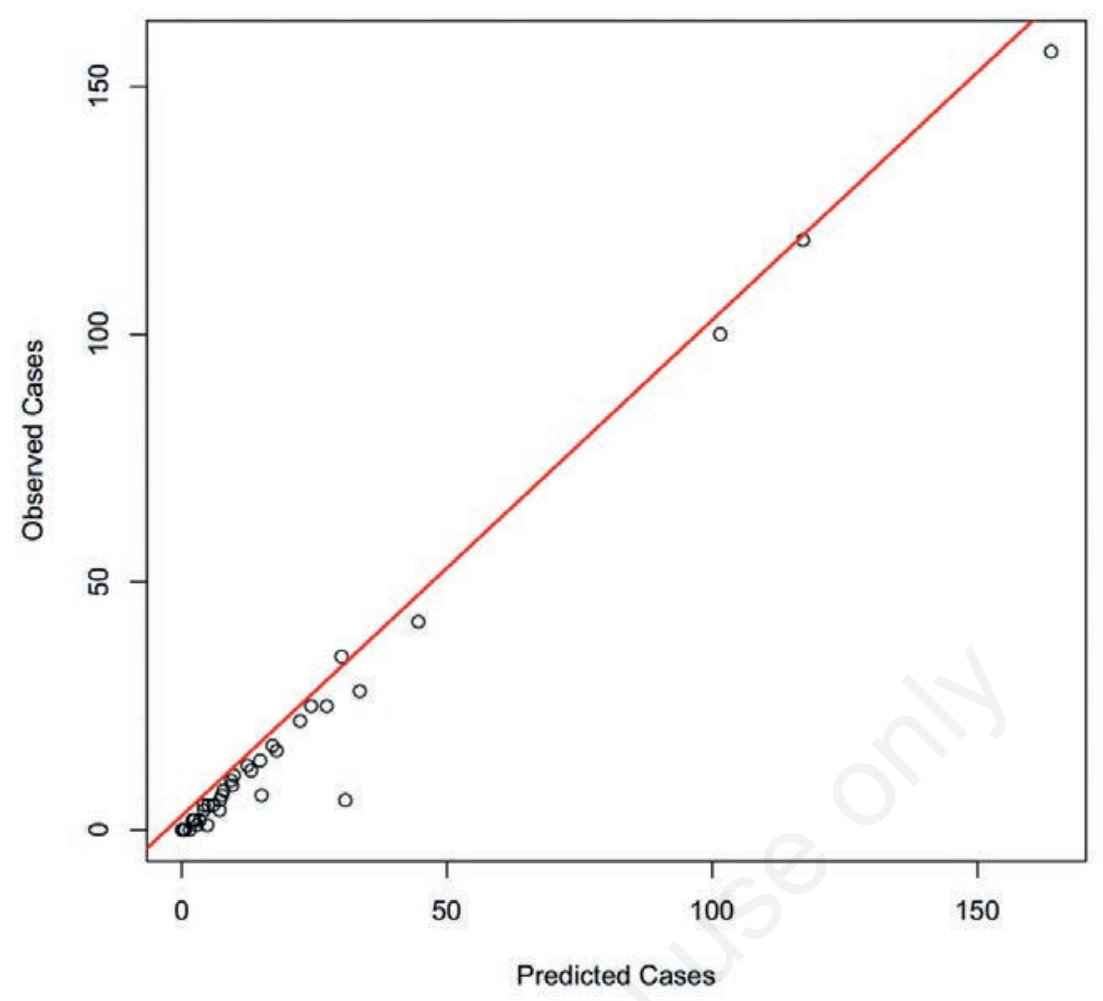

Figure 3. Plot of the observed heartworm infection cases in dogs from the laboratory data for the 37 southern Ontario census divisions against the predicted cases based on variables included in the spatial Generalized Linear Mixed Model Penalized Quasi-likelihood model.

There could also have been changes in heartworm risk for that census division, and dogs are now not exposed to the same level of heartworm risk as they were historically.

\section{Conclusions}

In conclusion, this study has shown that, overall, heartworm infection is a rare event in Ontario, but that geographic variations in heartworm risk exist. The geographic variation is strongly associated with variation in HDUs and CMI. Traditionally, the focus of heartworm testing has been southern Ontario, but heartworm is widespread among dogs in the province. It is not known if the spread of heartworm infection is endemic or related to travel; however, historically around $75 \%$ of heartworm infections were locally acquired (Slocombe, 1990). There was an increase in the number of regions in Ontario where heartworm infections were found from 2007 to 2016. It is possible that the increased testing in Ontario from 3,782 test results in 2007 to 136,581 test results in 2016 is likely due to increased concern regarding tick-borne pathogens, in both humans and dogs (Bouchard et al., 2015; PHO, 2018) and that this contributed to more heartworm infections being detected. Overall, the combination of increased testing over the study period and climate change may explain the heartworm infection clusters and the change in distribution seen in Ontario over 2007 to 2016.

\section{References}

Alho AM, Landum M, Ferreira C, Meireles J, Gonçalves L, de Carvalho LM, Belo S, 2014. Prevalence and seasonal variations of canine dirofilariosis in Portugal. Vet Parasitol 206:99105.

Assunção RM, Reis EA, 1999. A new proposal to adjust for Moran's I for population density. Stat Med 18:2147-62.

Berke O, 2004. Exploratory disease mapping: kriging the spatial risk function from regional count data. Int $\mathrm{J}$ Health Geogr 3:18-29.

Berke O, Waller L, 2010. On the effect of diagnostic misclassification bias on the observed spatial pattern in regional count data - a case study using West Nile virus mortality data from Ontario, 2005. Spat Spatiotemporal Epidemiol 1:117-22.

Beyer K, Tiwari C, Rushton G, 2012. Five essential properties of disease maps. Ann Assoc Am Geogr 102:1067-75.

Bouchard C, Leonard E, Koffi JK, Pelcat Y, Peregrine A, Chilton N, Rochon K, Lysyk T, Lindsay R, Ogden NH, 2015. The increasing risk of Lyme disease in Canada. Can Vet J 56:693-9.

Brown HE, Harrington LC, Kaufman PE, McKay T, Bowman DD, Nelson CT, Wang D, Lund R, 2012. Key factors influencing canine heartworm, Dirofilaria immitis, in the United States. Parasite Vectors 5:245-54.

Ciucă L, Musella V, Miron LD, Maurelli MP, Cringoli G, Bosco A, 
Rinaldi L, 2016. Geographic distribution of canine heartworm (Dirofilaria immitis) infection in stray dogs of eastern Romania. Geospat Health 11:318-23.

DMTI Spatial Inc., 2018. CanMap Postal Code Suite, 2018. Monograph on the Internet. Markham, Ontario: DMTI Spatial Inc. c2007-2014. Available from: http://geo1.scholarsportal. info/\#r/details/_uri@=3023771738 Accessed: March 1, 2018.

Dohoo IR, Martin SW, Stryhn H, 2003. Linear regression. In: Veterinary epidemiologic research. 1st ed. AVC Inc., Prince Edward Island, Canada.

Ernst J, Slocombe JOD, 1984. Mosquito vectors of Dirofilaria immitis in southwestern Ontario. Can J Zool 62:212-6.

ESRI, 2011. ArcGIS Desktop: Release 10. Redlands, CA: Environmental Systems Research Institute.

Fortin JF, Slocombe JOD, 1981. Temperature requirements for the development of Dirofilaria immitis in Aedes triseriatus and Ae. Vexans. Mosq News 41:625-35.

Genchi C, Mortarino M, Rinaldi L, Cringoli G, Traldi G, Genchi M, 2011. Changing climate and changing vector-borne disease distribution: the example of Dirofilaria in Europe. Vet Parasitol 176:295-9.

Graham W, Rubin SB, Boeckh A, Burhardt LF, Jones S, Miller M, Payne P, Rehm C, Smith-Blackmore M, Stannard R, Nelson CT, Atkins C, Carithers D, McCall J, von Simson C, 2012. Current canine guidelines for the diagnosis, prevention, and management of heartworm (Dirofilaria immitis) infection in dogs. Am Heartworm Society 1-32.

Greer A, Ng V, Fisman D, 2008. Climate change and infectious diseases in North America: the road ahead. Can Med Assoc J 178:715-22.

Herrin BH, Peregrine AS, Goring J, Beall MJ, Little SE, 2017. Canine infection with Borrelia burgdorferi, Dirofilaria immitis, Anaplasma spp. and Ehrlichia spp. in Canada, 2013-2014. Parasite Vectors 10:244-53.

IDEXX, 2016. SNAP 4Dx Plus Test. Available from: https://idexxcom-live-b02da1e51e754c9cb292133b9c56c33.aldryn-media.com/filer_public/9f/5e/9f5eeaf3-99b4436b-951f-cc0c27620676/snap-4dx-plus-test-accuracy.pdf Accessed: April 21, 2018.

Klotins KC, Martin SW, Bonnett BN, Peregrine AS, 2000. Canine heartworm testing in Canada: Are we being effective? Can Vet J 41:929-37.

Knight DH, Lok JB, 1998. Seasonality of heartworm infection and implications for chemoprophylaxis. Clin Tech Small An P 13:77-82.

Kulldorff M, Huang L, Konty K, 2009. A scan statistic for continuous data based on the normal probability model. Int J Health Geo 8:58-67.

Ledesma N, Harrington L, 2011. Mosquito vectors of dog heartworm in the United States: vector status and factors influencing transmission efficiency. Top Companion Anim M 26:17885.

Ludlam KW, Jachowski LA, Ottawa GF, 1970. Potential vectors of Dirofilaria immitis. J Am Vet Med Assoc 157:1354-9.

McPherson M, García-García A, Cuesta-Valero FJ, Beltrami H, Hansen-Ketchum P, MacDougall D, Ogden NH, 2017. Expansion of the Lyme disease vector Ixodes scapularis in Canada inferred from CMIP5 climate projections. Environ Health Perspect 125:1-9.
Morchón R, Carretón E, González-Miguel J, Mellado-Hernández I, 2012. Heartworm disease (Dirofilaria immitis) and their vectors in Europe - new distribution trends. Front Physiol 3:1-11.

Natural Resources Canada, 2018. Natural Resources Canada. Regional, national and international climate modeling. Available from: https://cfs.nrcan.gc.ca/projects/3 Accessed: February 12, 2018.

PHO, 2018. Public Health Ontario. Lyme disease. Available from: https://www.publichealthontario.ca/en/BrowseByTopic/Infecti ousDiseases/Pages/IDLandingPages/Lyme-Disease.aspx Accessed: July 2018.

R Core Team, 2017. R: A language and environment for statistical computing. R Foundation for Statistical Computing, Vienna, Austria. Available from: https://www.R-project.org/ Accessed: April 2018.

RStudio Team, 2016. RStudio: Integrated development tool for R. Available from: http://www.rstudio.com/ Accessed: April 2018.

Sassnau R, Daugschies A, Lendner M, Genchi C, 2014. Climate suitability for the transmission of Dirofilaria immitis and $D$. repens in Germany. Vet Parasitol 205:239-45.

SaTScan, 2018. Software for the Spatial and Space-Time Scan Statistics. Kulldorff M. Information Management Services Inc., Calverton, MA. Available from: https://www.satscan.org/ Accessed: April 2018.

Slocombe JOD, Surgeoner GA, Srivastiva B, 1989. Determination of the heartworm transmission period and its use in diagnosis and control. Epizootiology 19-26.

Slocombe JOD, 1990. Heartworm in dogs in Canada in 1989. Can Vet J 31:499-505.

Slocombe JOD, 2011. Heartworm in Canada in 2010 with comments on Ontario. Available from: http://ovc.uoguelph. ca/doc/heartworm/2010/Quebec_en.pdf Accessed January, 2018.

Statistics Canada, 2011. Population and dwelling counts, for Canada, provinces and territories, census divisions, and census subdivisions (municipalities), 2011 and 2006 censuses (table). Population and Dwelling Count Highlight Tables. 2011 Census. Available from: http://www12.statcan.gc.ca/censusrecensement/2011/dp-pd/hlt-fst/pd-pl/File.cfm?T=304\&SR $=1 \& \mathrm{RPP}=9999 \& \mathrm{PR}=35 \& \mathrm{CMA}=0 \& \mathrm{~S}=51 \& \mathrm{O}=\mathrm{A} \& \mathrm{LANG}=\mathrm{En}$ g\&OFT $=$ CSV Accessed: March 17, 2018.

Statistics Canada, 2016. Statistics Canada 2011 Census - Boundary files. Available from: https://www12.statcan.gc.ca/censusrecensement/2011/geo/bound-limit/bound-limit-2011-eng.cfm Accessed: March 14, 2018.

Tango T, Takahashi K, 2012. A flexible spatial scan statistic with a restricted likelihood ratio for detecting disease clusters. Stat Med 31:4201-18.

Vezzani D, Carbajo AE, 2006. Spatial and temporal transmission risk of Dirofilaria immitis in Argentina. Int J Parasitol 36:1463-72.

Wang D, Bowman DD, Brown HE, Harrington LC, Kaufman P, McKay T, Nelson CT, Sharp JL, Lund R, 2014. Factors influencing U.S. canine heartworm (Dirofilaria immitis) prevalence. Parasite Vectors 7:264-82.

Waller LA, Gotway CA, 2004. Applied spatial statistics for public health data. 1st ed. John Wiley \& Sons, New Jersey, United States. pp 227-234. 